\title{
The Analysis of Elliptic Families. I. Metrics and Connections on Determinant Bundles
}

\author{
Jean-Michel Bismut ${ }^{1}$ and Daniel S. Freed ${ }^{2}$ \\ 1 Département de Mathématique, Université Paris-Sud, Bâtiment 425, F-91405 Orsay, France \\ 2 Department of Mathematics, M.I.T., Cambridge, MA 02139, USA
}

\begin{abstract}
In this paper, we construct the Quillen metric on the determinant bundle associated with a family of elliptic first order differential operators. We also introduce a unitary connection on $\lambda$ and calculate its curvature. Our results will be applied to the case of Dirac operators in a forthcoming paper.
\end{abstract}

In [Q2], Quillen gave a construction of a metric and of a holomorphic connection on the determinant bundle of a family of $\bar{\partial}$ operators. On the other hand, Bismut gave in [B1] a heat equation proof of the Atiyah-Singer Index Theorem for families of Dirac operators [AS1] using the superconnection formalism of Quillen [Q1]. In this paper, we extend the construction of Quillen [Q2] to the case of an arbitrary family of first order elliptic differential operators.

More precisely, let $\stackrel{\text { Z }}{\rightarrow} B$ be a compact fibering of manifolds and let $D_{+}$be a family of first order elliptic differential operators. $D_{+}$can be considered as a smooth section of $\operatorname{Hom}\left(H_{+}^{\infty}, H_{-}^{\infty}\right)$, where $H_{+}^{\infty}, H_{-}^{\infty}$ are infinite dimensional Hermitian bundles over $B$. If $\lambda$ is the line bundle $\left(\operatorname{det} \operatorname{Ker} D_{+}\right)^{*} \otimes\left(\operatorname{det} \operatorname{Coker} D_{+}\right)$, we construct a metric and a unitary connection on $\lambda$, and we calculate the corresponding curvature.

To explain the construction, let us temporarily assume that $H_{+}^{\infty}, H_{-}^{\infty}$ are instead finite dimensional Hermitian bundles over $B$ which have the same dimension. In this case $\lambda$ can be identified with $\left(\operatorname{det} H_{+}^{\infty}\right)^{*} \otimes \operatorname{det} H_{-}^{\infty}$, and so is naturally endowed with a Hermitian metric $\|\cdot\|$. Clearly $\operatorname{det} D_{+}$is a section of $\lambda$.

Let $D_{-}$be the adjoint of $D_{+}$, and set

$$
H^{\infty}=H_{+}^{\infty} \oplus H_{-}^{\infty} ; \quad D=\left[\begin{array}{cc}
0 & D_{-} \\
D_{+} & 0
\end{array}\right] .
$$

Then

$$
\left\|\operatorname{det} D_{+}\right\|=\left[\operatorname{det} D_{-} D_{+}\right]^{1 / 2}=\left[\operatorname{det} D^{2}\right]^{1 / 4} .
$$

Also if $H_{+}^{\infty}, H_{-}^{\infty}$ are endowed with a unitary connection $\tilde{\nabla}^{u}, \lambda$ is also endowed with a unitary connection ${ }^{1} \nabla$. Where $D_{+}$is invertible, we have for $Y \in T B$,

$$
\tilde{\nabla}_{Y}^{u} \operatorname{det} D_{+}=\operatorname{det} D_{+} \operatorname{Tr}\left[D_{+}^{-1} \tilde{\nabla}_{Y}^{u} D_{+}\right] .
$$

By [Q1], the graded algebra End $H^{\infty}$ is endowed with a trace $\operatorname{Tr}$ and a supertrace 
$\operatorname{Tr}_{s}$. We rewrite $(0.3)$ in the form

$$
\frac{\tilde{\nabla}_{Y}^{u} \operatorname{det} D_{+}}{\operatorname{det} D_{+}}=\frac{1}{2}\left\{\operatorname{Tr}\left[D^{-1} \tilde{\nabla}_{Y}^{u} D\right]+\operatorname{Tr}_{s}\left[D^{-1} \tilde{\nabla}_{Y}^{u} D\right]\right\} .
$$

Also since $D$ is self-adjoint, $\operatorname{Tr}\left[D^{-1} \tilde{\nabla}_{Y}^{u} D\right]$ is real, and $\operatorname{Tr}_{s}\left[D^{-1} \tilde{\nabla}_{Y}^{u} D\right]$ is purely imaginary. Finally observe that

$$
d \log \left\|\operatorname{det} D_{+}\right\|=\frac{1}{2} \operatorname{Tr}\left[D^{-1} \tilde{\nabla}^{u} D\right] .
$$

Equations (0.3)-(0.5) fully suggest how to define a metric and a connection when $H_{+}^{\infty}, H_{-}^{\infty}$ are infinite dimensional. In fact in [Q1], Quillen used the zêta function renormalization of the determinant to define the metric \| \|. This is also what we do here. In the right-hand side of $(0.4)$, we should now make sense of $\operatorname{Tr}_{s}\left[D^{-1} \tilde{\nabla}^{u} D\right]$. The idea is to use a heat equation-or zêta function-renormalization and so define formally,

$$
\operatorname{Tr}_{s}\left[D^{-1} \tilde{\nabla}^{u} D\right]=F p\left(\operatorname{Tr}_{s}\left[\exp \left(-t D^{2}\right) D^{-1} \tilde{\nabla}^{u} D\right]\right),
$$

where $F p$ is an adequately defined finite part of the right-hand side of $(0.6)$ as $t \downarrow \downarrow 0$.

The real miracle is that the right-hand side of $(0.6)$ naturally appears when transgressing in the most natural way the heat equation formula for the Chern character $\operatorname{ch}\left(\operatorname{Ker} D_{+}-\operatorname{Ker} D_{-}\right)$obtained in Bismut [B1, Sect.2] by using the superconnection formalism of Quillen [Q1].

Our paper is organized in the following way. In a), we describe the fibered manifold $M \stackrel{Z}{\rightarrow} B$. In b), we introduce the unitary connection $\tilde{\nabla}^{u}$ on the infinite dimensional bundle $H^{\infty}$. In c), using [B1, Sect.2], we prove the analogue of the results of Atiyah-Bott-Patodi [ABP], i.e. express $\operatorname{ch}\left(\operatorname{Ker} D_{+}-\operatorname{Ker} D_{-}\right)$in terms of certain asymptotic expansions. In d), we transgress Quillen's superconnections so that the right-hand side of $(0.6)$ appears naturally. In e), we calculate asymptotic expansions related to the right-hand side of (0.6). In $\mathrm{f}$ ), we describe the determinant bundle $\lambda$ as in [Q2].

In $\mathrm{g}$ ), we construct the Quillen metric on $\lambda$. In fact we here consider a family of metrics because of certain scaling discrepancies. In $h$ ), we calculate what will later be the connection forms of $\lambda$.

In i), we prove a key additivity property of Quillen's superconnections. This permits us in $\mathrm{j}$ ) to construct the connection ${ }^{1} \nabla$ on $\lambda$ and to calculate its curvature. Finally in k), we prove that in a product situation, ${ }^{1} \nabla$ is holomorphic. This extends the results of Quillen [Q2].

In [BF2], we will apply our results to the case of a family of Dirac operators. A future paper by Freed [F] will discuss geometric and topological aspects of this work and give many examples, particularly related to anomalies. The results contained in this paper have been announced in [BF1].

\section{a) Description of the Fibered Manifold}

$n=2 l$ is an even integer, and $m$ is an integer. $M, B$ are smooth manifolds of dimension $n+m$ and $m . g_{B}$ denotes a metric on $T B . Z$ is a compact connected 
manifold of dimension $n$, which we assume to be orientable and spin. $\pi: M \underset{Z}{B} B$ is a fibration of $M$ on $B$, which is modelled on $Z$. There is then an open covering $\mathscr{U}$ of $B$ such that if $U \in \mathscr{U}, \pi^{-1}(U)$ is diffeomorphic to $U \times Z$. For $y \in B, Z_{y}$ is the fiber $\pi^{-1}(\{y\})$.

We assume that $T Z$ is oriented and spin. Let $g_{Z}$ be a metric on $T Z . O$ denotes the $\mathrm{SO}(n)$ bundle of oriented orthonormal frames in $T Z, O^{\prime}$ a Spin $(n)$ bundle which lifts $O$ such that $O^{\prime} \underset{\sigma}{\rightarrow} O$ induces the covering projection $\operatorname{Spin}(n) \rightarrow \mathrm{SO}(n)$ on each fiber.

$\operatorname{Spin}(n)$ acts unitarily on the vector space of spinors $S=S_{+} \oplus S_{-}$. Let $F=$ $F_{+} \oplus F_{-}$be the Hermitian bundles of spinors, $F=O^{\prime} \times_{\operatorname{Spin}(n)} S, F_{ \pm}=O^{\prime} \times_{\operatorname{Spin}(n)}$ $S_{ \pm}$. Let $T^{H} M$ be a smooth subbundle of $T M$ such that

$$
T M=T^{H} M \oplus T Z .
$$

$T^{H} M, T Z$ are the horizontal and vertical parts of $T M$. Let $P_{Z}$ be the projection operator of $T M$ on $T Z$ associated with the splitting (1.1).

We identify $T^{H} M$ and $\pi^{*} T B . T^{H} M$ inherits the scalar product $g_{B}$ of $T B$. We denote by $g_{B} \oplus g_{Z}$ the metric of $T M$, which coincides with $g_{B}$ on $T B$, with $g_{Z}$ on $T Z$ and is such that $T^{H} M$ and $T Z$ are orthogonal.

Let $\nabla^{L}$ be the Levi-Civita connection on $T M$.

Definition 1.1. $\nabla$ denote the connection on $T Z$

$$
\nabla=P_{Z} \nabla^{L}
$$

In [B1, Theorem 1.9], it is proved that $\nabla$ does not depend on $g_{B} . \nabla$ lifts naturally into a unitary connection on $F_{ \pm}$. $\xi$ is a Hermitian bundle on $M$, which is endowed with a unitary connection, which we also note $\nabla$. The Hermitian bundles $F_{ \pm} \otimes \xi$ are then naturally endowed with a unitary connection $\nabla$.

\section{b) Connections on Infinite Dimensional Bundles}

$H^{\infty}=H_{+}^{\infty} \oplus H_{-}^{\infty}$ denotes the set of $C^{\infty}$ sections of $F \otimes \xi=\left(F_{+} \otimes \xi\right) \oplus\left(F_{-} \otimes \xi\right)$ over $M$. As in [B1], we will consider $H^{\infty}, H_{ \pm}^{\infty}$ as being the sets of $C^{\infty}$ sections of infinite dimensional bundles over $B$, whose fibers $H_{y}^{\infty}, H_{ \pm, y}^{\infty}$ are the sets of $C^{\infty}$ sections of $F \otimes \xi, F_{ \pm} \otimes \xi$ over the fiber $Z_{y}$.

For $s \in R, y \in B$, let $H_{y}^{s}, H_{ \pm, y}^{s}$ be the set of sections of $F \otimes \xi, F_{ \pm} \otimes \xi$ over $Z_{y}$, which belong to the $s$-Sobolev space. Contrary to $H^{\infty}, H^{s}$ is not a smooth bundle over $B$, but is only continuous.

Let $d x$ be the Riemannian volume element in the fibers $Z . H_{y}^{\infty}$ is naturally endowed with the Hermitian product,

$$
h, h^{\prime} \in H_{y}^{\infty} \rightarrow\left\langle h, h^{\prime}\right\rangle=\int_{Z_{y}}\left\langle h, h^{\prime}\right\rangle(x) d x .
$$

For $y \in T B$, let $Y^{H}$ be the lift of $Y$ in $T^{H} M$, so that

$$
Y^{H} \in T^{H} M, \quad \pi_{*} Y^{H}=Y .
$$

We now define a connection on $H_{ \pm}^{\infty}$ as in [B1, Definition 1.10]. 
Definition 1.2. $\tilde{\nabla}$ denotes the connection on $H^{\infty}$ which is such that if $h \in H^{\infty}$,

$$
\tilde{\nabla}_{Y} h=\nabla_{Y^{H}} h .
$$

By [B1, Proposition 1.11], the curvature $\tilde{R}$ of $\tilde{\nabla}$ is a first order differential operator acting fiberwise. Although $\nabla$ is unitary on $F \otimes \xi, \tilde{\nabla}$ is in general not unitary on $H^{\infty}$, since the volume element $d x$ is not invariant under the holonomy group of the connection $\tilde{\nabla}$. However a mild modification of $\tilde{\nabla}$ makes the new connection unitary.

In fact, let $Y$ be a smooth vector field on $B . Y^{H}$ acts on the fibration $Z$, and in particular on the volume element $d x$ of $Z$. For any $x \in M$, the divergence $\operatorname{div}_{Z}\left(Y^{H}\right)$ of $Y^{H}$ with respect to $d x$ is well defined. One readily verifies that $Y \rightarrow \operatorname{div}_{Z}\left(Y^{H}\right)(x)$ is a tensor.

Definition 1.3. $k$ denotes the smooth vector field in $T^{H} M$ such that for any $Y \in T B$

$$
\operatorname{div}_{Z} Y^{H}(x)=2\left\langle k, Y^{H}\right\rangle(x) \text {. }
$$

$\tilde{\nabla}$ is the connection on $H^{\infty}$ defined by the relation

$$
\tilde{\nabla}_{Y}^{u}=\tilde{\nabla}_{Y}+\left\langle k, Y^{H}\right\rangle \text {. }
$$

Proposition 1.4. The connection $\tilde{\nabla}^{u}$ is unitary on $H^{\infty}$.

Proof. If $h, h^{\prime} \in H^{\infty}$, we have the relation

$$
Y \int_{Z}\left\langle h, h^{\prime}\right\rangle(x) d x=\int_{Z}\left(\left\langle\tilde{\nabla}_{Y} h, h^{\prime}\right\rangle+\left\langle h, \tilde{\nabla}_{Y} h^{\prime}\right\rangle+\operatorname{div}_{Z}\left(Y^{H}\right)\left\langle h, h^{\prime}\right\rangle\right) d x .
$$

The Proposition is now obvious.

c) Quillen's Superconnections and the Chern Character of $\operatorname{Ker} D_{+}-\operatorname{Ker} D_{-}$

$D_{+, y}$ is a smooth family of first order elliptic differential operators acting fiberwise on $Z$, which sends $H_{+, y}^{\infty}$ into $H_{-, y}^{\infty}$. $D_{-, y}$ denotes the formal adjoint of $D_{+, y}$ with respect to the Hermitian product (1.3). $D_{y}$ is the operator acting on $H_{y}^{\infty}=H_{+, y}^{\infty} \oplus H_{-, y}^{\infty}$,

$$
D_{y}=\left[\begin{array}{cc}
0 & D_{-, y} \\
D_{+, y} & 0
\end{array}\right] \text {. }
$$

$H^{\infty}=H_{+}^{\infty} \oplus H_{-}^{\infty}$ is a $Z_{2}$ graded vector bundle over $B$. Let $\tau$ be the involution of $H^{\infty}$ defining the grading, i.e. $\tau= \pm 1$ on $H_{ \pm}^{\infty}$. End $H^{\infty}$ is naturally $Z_{2}$ graded, the even (respectively odd) elements of End $H^{\infty}$ commuting (respectively anticommuting) with $\tau$. If $A$ is a trace class operator acting on $H_{y}^{\infty}$, we define its supertrace $\operatorname{Tr}_{s} A$ by the relation

$$
\operatorname{Tr}_{s} A=\operatorname{Tr} \tau A .
$$

End $H_{y}^{\infty} \hat{\otimes} \Lambda\left(T_{y}^{*} B\right)$ is also $Z_{2}$ graded. We extend $\operatorname{Tr}$, $\operatorname{Tr}_{s}$ to trace class elements $A^{\prime}$ in End $H_{y}^{\infty} \hat{\otimes} \Lambda\left(T_{y}^{*} B\right)$. $\operatorname{Tr} A^{\prime}, \operatorname{Tr}_{s} A^{\prime}$ are now in $\Lambda\left(T_{y}^{*} B\right)$. We use the convention that if $\omega \in \Lambda\left(T^{*} B\right)$,

$$
\operatorname{Tr}\left[\omega A^{\prime}\right]=\omega \operatorname{Tr}\left[A^{\prime}\right], \operatorname{Tr}_{s}\left[\omega A^{\prime}\right]=\omega \operatorname{Tr}_{s}\left[A^{\prime}\right]
$$


For any $t>0, \tilde{\nabla}^{u}+\sqrt{ } t D$ is a superconnection on $H^{\infty}$ in the sense of Quillen [Q1]. By [B1, Sect. 2] $\left(\tilde{\nabla}^{u}+\sqrt{ } t D\right)^{2}$ is an elliptic second order differential operator acting fiberwise, which is even if End $H^{\infty} \hat{\otimes} \Lambda\left(T^{*} B\right)$.

$\exp -\left(\tilde{\nabla}^{u}+\sqrt{ } t D\right)^{2}$ is then even in End $H^{\infty} \hat{\otimes} \Lambda\left(T^{*} B\right)$ and is given by a $C^{\infty}$ kernel $T_{t}\left(x, x^{\prime}\right)$ along the fibers $Z$. By noting that $T_{t}(x, x)$ is even in $\operatorname{End}(F \otimes \xi)_{x} \hat{\otimes} \Lambda_{\pi(x)}\left(T^{*} B\right)$, and using the convention (1.11), $\operatorname{Tr}_{s}\left[T_{t}(x, x)\right]$ is an even element of $\Lambda\left(T_{\pi x}^{*} B\right)$.

If $E$ is a complex vector bundle over $B$ endowed with a smooth connection whose curvature is $L$, set

$$
\operatorname{ch}_{1} E=\operatorname{Tr} \exp -L .
$$

$\operatorname{ch}_{1} E$ is a scaled representative of the Chern character of $E$. If $\omega \in \Lambda\left(T^{*} B\right)$, $\omega^{(j)}$ denotes the component of $\omega$ in $\Lambda^{j}\left(T^{*} B\right)$. If $B$ is compact, by [AS1], $\operatorname{Ker} D_{+}-\operatorname{Ker} D_{-}$is a well-defined element of $K(B)$.

We first state a general result which is the natural extension of Atiyah-Bott-Patodi [ABP].

Theorem 1.5. For any $t>0$, the $C^{\infty}$ differential form over $B$

$$
\operatorname{Tr}_{s} \exp -\left(\tilde{\nabla}^{u}+\sqrt{ } t D\right)^{2}=\int_{Z} \operatorname{Tr}_{s}\left[T_{t}(x, x)\right] d x
$$

is closed and its cohomology class does not depend on $t$. If $B$ is compact, it represents in cohomology $\mathrm{ch}_{1}\left(\operatorname{Ker} D_{+}-\operatorname{Ker} D_{-}\right)$. As $t \downarrow \downarrow 0$, for any $k \in N$,

$$
\operatorname{Tr}_{s} \exp -\left(\tilde{\nabla}^{u}+\sqrt{ } t D\right)^{2}=\sum_{-n / 2-[m / 2]}^{k} a_{j}(y) t^{j}+o\left(t^{k}, y\right),
$$

where $\left(a_{j}\right)$ are $C^{\infty}$ differential forms on $B$, and $o\left(t^{k}, y\right)$ is uniform on compact sets in $B$. For p even (respectively odd) $a_{j}^{(2 p)}$ is real (respectively purely imaginary). For $j \neq 0, a_{j}$ is exact. $a_{0}$ is closed and is in the same cohomology class as (1.13). If $B$ is compact, $a_{0}$ represents in cohomology $\mathrm{ch}_{1}\left(\operatorname{Ker} D_{+}-\operatorname{Ker} D_{-}\right)$.

$$
\text { For } 0 \leqq p \leqq[m / 2], \quad j<-n / 2-p, \quad a_{j}^{(2 p)}=0 .
$$

Proof. The first part of the Theorem is proved in [B1, Theorem 2.6] when $\tilde{\nabla}^{u}$ is replaced by $\tilde{\nabla}$, and follows from [B1, Proposition 2.10] in general.

Also by Greiner [Gr, Theorem 1.6.1], for every $y \in B$, we have the asymptotic expansion

$$
\operatorname{Tr}_{s} \exp -t\left(\tilde{\nabla}^{u}+D\right)^{2}=\sum_{-n / 2}^{k^{\prime}} a_{j}^{\prime}(y) t^{j}+o\left(t^{k^{\prime}}, y\right) .
$$

In (1.15), $\left(a_{j}^{\prime}\right)$ are $C^{\infty}$ differential forms on $B$, and $o\left(t^{k^{\prime}}, y\right)$ is uniform on compact subsets of $B$, because the fibers $Z$ are compact.

Let $\varphi_{t}$ be the homomorphism from $\Lambda\left(T^{*} B\right)$ into itself which to a one form $\omega$ associates $\omega / \sqrt{ } t$. Clearly

$$
\operatorname{Tr}_{s} \exp -\left(\tilde{\nabla}^{u}+\sqrt{ } t D\right)^{2}=\varphi_{t}\left[\operatorname{Tr}_{s} \exp -t\left(\tilde{\nabla}^{u}+D\right)^{2}\right] .
$$


Also for $0 \leqq p \leqq[m / 2]$,

$$
\varphi_{t}\left(o\left(t^{k^{\prime}}, y\right)\right)^{(2 p)}=o\left(t^{k^{\prime}-p}, y\right) .
$$

By choosing $k^{\prime}$ large enough, we obtain (1.14). The final statement in the theorem also follows from (1.15), (1.16).

Let $\psi$ be the linear mapping from End $H^{\infty} \hat{\otimes} \Lambda\left(T^{*} B\right)$ into itself which to $B=A$ $d y^{\alpha_{1}} \ldots d y^{\alpha_{p}}$ associates $B^{\prime}=d y^{\alpha_{p}} \cdots d y^{\alpha_{1}} A^{*}$, where $A^{*}$ is the formal adjoint of $A$. Clearly

$$
\left(\tilde{\nabla}^{u}+\sqrt{ } t D\right)^{2}=\left(\tilde{\nabla}^{u}\right)^{2}+\sqrt{ } t\left(\tilde{\nabla}^{u} D\right)+t D^{2} .
$$

Since $\tilde{\nabla}^{u}$ is unitary, its curvature $\left(\tilde{\nabla}^{u}\right)^{2}$ takes values in skew-adjoint elements of End $H^{\infty}$. Also since $D$ is self-adjoint, for any $Y \in T B, \widetilde{\nabla}_{Y}^{u} D$ is self-adjoint. We then find that

$$
\psi\left(\tilde{\nabla}^{u}\right)^{2}=\left(\tilde{\nabla}^{u}\right)^{2} ; \quad \psi\left(\tilde{\nabla}^{u} D\right)=-\tilde{\nabla}^{u} D ; \quad \psi\left(D^{2}\right)=D^{2},
$$

and so

$$
\psi\left(\tilde{\nabla}^{u}+\sqrt{ } t D\right)^{2}=\left(-\tilde{\nabla}^{u}+\sqrt{ } t D\right)^{2} .
$$

We then obtain

$$
\psi\left(\exp -\left(\tilde{\nabla}^{u}+\sqrt{ } t D\right)^{2}\right)=\exp -\left(-\tilde{\nabla}^{u}+\sqrt{ } t D\right)^{2} .
$$

Since $\operatorname{Tr}_{s}$ vanishes on odd element of End $H^{\infty}$, we get

$$
\operatorname{Tr}_{s} \psi\left(\exp -\left(\tilde{\nabla}^{u}+\sqrt{ } t D\right)^{2}\right)=\operatorname{Tr}_{s} \exp -\left(\tilde{\nabla}^{u}+\sqrt{ } t D\right)^{2} .
$$

If $A$ is trace class, clearly $\operatorname{Tr}_{s} A^{*}=\overline{\operatorname{Tr}_{s} A}$. From (1.21), we find that if $p$ is even (respectively odd) $\left[\operatorname{Tr}_{s} \exp -\left(\widetilde{\nabla}^{u}+\sqrt{ } t D\right)^{2}\right]^{(2 p)}$ is real (respectively purely imaginary). The corresponding statement on $\left(a_{j}\right)$ follows.

Let $c$ be a $C^{\infty}$ cycle in $B$ (so that $\partial c=0$ ). Clearly, by the first part of the theorem,

$$
\int_{c} \operatorname{Tr}_{s} \exp -\left(\tilde{\nabla}^{u}+\sqrt{ } t D\right)^{2}
$$

does not depend on $t$. Using (1.14), we find that $\int_{c} a_{0}$ coincides with (1.22) and also that $\int_{c} a_{j}=0$ for $j \neq 0$. The theorem is proved.

Remark 1 . The family $D$ gives a map from $B$ into the classifying space $Z \times B U_{\infty}$. In general, the differential forms (1.13) and $a_{0}$ represent in cohomology the pull back of cohomology classes on $Z \times B U_{\infty}$ through the mapping $D$. This is proved in Theorem 1.5 when $B$ is compact, and follows from Theorem 1.5 in general by restriction to compact pieces in $B$. The results which follow will be true for any parametrizing manifold $B$.

\section{d) Transgression of the Chern Character}

For $s>0, e^{-s D^{2}}$ is given by a $C^{\infty}$ kernel on $Z \cdot e^{-s D^{2}} \tilde{\nabla}^{u} D D$ is then a 1 -form on $B$ with values in trace class elements of End $H^{\infty}$. 
We now prove a fundamental transgression formula.

Theorem 1.6. For $0<t<T<+\infty$, the following identity holds:

$$
\begin{gathered}
{\left[\operatorname{Tr}_{s} \exp -\left(\tilde{\nabla}^{u}+\sqrt{ } t D\right)^{2}\right]^{(2)}-\left[\operatorname{Tr}_{s} \exp -\left(\tilde{\nabla}^{u}+\sqrt{ } T D\right)^{2}\right]^{(2)}} \\
=-\frac{d}{2} \int_{t}^{T} \operatorname{Tr}_{s}\left[\exp \left(-s D^{2}\right) \tilde{\nabla}^{u} D D\right] d s .
\end{gathered}
$$

Proof. We will do formal computations, which are justifiable using $C^{\infty}$ kernels as in [B1, Sect. 2].

By proceeding as in [B1, Proposition 2.10 and Remark 2.3],

$$
\frac{\partial}{\partial s} \operatorname{Tr}_{s}\left[\exp -\left(\tilde{\nabla}^{u}+s D\right)^{2}\right]=-d \operatorname{Tr}_{s}\left[D \exp -\left(\tilde{\nabla}^{u}+s D\right)^{2}\right]
$$

and so

$$
\left.\frac{\partial}{\partial s} \operatorname{Tr}_{s} \exp -\left(\tilde{\nabla}^{u}+s D\right)^{2}\right]^{(2)}=-d\left[\operatorname{Tr}_{s} D \exp -\left(s \tilde{\nabla}^{u} D+s^{2} D^{2}\right)\right]^{(1)}
$$

By Duhamel's formula we have

$$
\begin{aligned}
\exp -\left(s \tilde{\nabla}^{u} D+s^{2} D^{2}\right)= & \exp -\left(s^{2} D^{2}\right)-\int_{0}^{1} \exp -v\left(s \tilde{\nabla}^{u} D+s^{2} D^{2}\right) \\
& \cdot s \tilde{\nabla}^{u} D \exp \left(-(1-v) s^{2} D^{2}\right) d v
\end{aligned}
$$

Now $\tilde{\nabla}^{u} D$ being of degree 1 in $\Lambda\left(T^{*} B\right)$, we have

$$
\left[\exp -\left(s \tilde{\nabla}^{u} D+s^{2} D^{2}\right)\right]^{(1)}=-\int_{0}^{1} \exp \left(-v s^{2} D^{2}\right) s \tilde{\nabla}^{u} D \exp \left(-(1-v) s^{2} D^{2}\right) d v
$$

Since by [Q1], $\operatorname{Tr}_{s}$ vanishes on supercommutators, we get

$$
\left[\operatorname{Tr}_{s} D \exp -\left(s \tilde{\nabla}^{u} D+s^{2} D^{2}\right)\right]^{(1)}=-s \operatorname{Tr}_{s}\left[\exp -s^{2} D^{2} \tilde{\nabla}^{u} D D\right] .
$$

Equation (1.23) follows from (1.24), (1.26).

\section{e) Asymptotic Expansions of Traces and Supertraces}

We now calculate certain asymptotics of traces and supertraces and in particular the small time expansions of both sides of (1.23).

Theorem 1.7. For any $t>0$, the function $\frac{1}{2} \operatorname{Tr}\left[\exp -t D^{2}\right]$ is real, and moreover

$$
d \frac{1}{2} \operatorname{Tr}\left[\exp -t D^{2}\right]=-t \operatorname{Tr}\left[\exp \left(-t D^{2}\right) \tilde{\nabla}^{u} D D\right] .
$$

There are $C^{\infty}$ real functions $A_{-n / 2} \cdots A_{j} \cdots$ on $B$, and $C^{\infty}$ purely imaginary 1 forms $B_{-n / 2} \cdots B_{j} \cdots$ on $B$ such that for any $k \in N$, as $t \downarrow \downarrow 0$,

$$
\begin{aligned}
& \frac{1}{2} \operatorname{Tr}\left[\exp \left(-t D^{2}\right)\right]=\sum_{-n / 2}^{k} A_{j} t^{j}+o\left(t^{k}, y\right), \\
& \operatorname{Tr}\left[\exp \left(-t D^{2}\right) \tilde{\nabla}^{u} D D\right]=-\sum_{-n / 2}^{k} d A_{j} t^{j-1}+o\left(t^{k-1}, y\right),
\end{aligned}
$$




$$
\operatorname{Tr}_{s}\left[\exp \left(-t D^{2}\right) \tilde{\nabla}^{u} D D\right]=-\sum_{-n / 2}^{k} B_{j} t^{j-1}+o\left(t^{k-1}, y\right)
$$

The various $o\left(t^{k-1}, y\right)$ are uniform on compact subsets of $B$. Also

$$
d B_{j}=-2 j a_{j}^{(2)}, \quad-n / 2 \leqq j<+\infty .
$$

In particular $d B_{0}=0$.

Proof. Equation (1.27) is trivial. The first line of (1.28) follows from [Gr, Theorem 1.6.1]. Using (1.27), and differentiating the right-hand side of (1.28) we obtain the third formula in (1.28). Using the same procedure as in (1.17)-(1.19), we find immediately that $\operatorname{Tr}_{s}\left[\exp -t D^{2} \tilde{\nabla}^{u} D D\right]$ is purely imaginary. Differentiating the parametrix of $e^{-t D 2}$ as in Greiner [Gr, Lemma 1.5.5], we obtain the third line in (1.28). Using (1.14), (1.28) and comparing the asymptotic expansions of both sides of (1.23), we obtain (1.29).

Remark 2. $A_{0}, B_{0}$ will play an important role in the sequel. We will prove in [BF2] that $B_{0}$ is exact.

We will use the following trivial identities;

$$
\begin{aligned}
& \operatorname{Tr}\left[e^{-t D_{+} D_{-}} \tilde{\nabla}^{u} D_{+} D_{-}\right]=-\operatorname{Tr}\left[e^{-t D_{-} D_{+}} D_{-} \tilde{\nabla}^{u} D_{+}\right]=\frac{1}{2} \operatorname{Tr}\left[e^{-t D^{2}} \tilde{\nabla}^{u} D D\right] \\
& -\frac{1}{2} \operatorname{Tr}_{s}\left[e^{-t D^{2}} \tilde{\nabla}^{u} D D\right] \text {. }
\end{aligned}
$$

Note that in the right-hand side of (1.30), the first term is real, and the second purely imaginary.

\section{f) The Determinant Line Bundle}

The determinant bundle of the elliptic family $D_{+}$is the bundle whose fiber $\lambda_{y}$ at $y \in B$ is

$$
\lambda_{y}=\left(\operatorname{det} \operatorname{Ker} D_{+, y}\right) * \otimes \operatorname{det}\left(\operatorname{Coker} D_{+, y}\right) .
$$

Since the dimension of $\operatorname{Ker} D_{+, y}$ may jump as $y$ varies, $\lambda$ is not yet a smooth line bundle. We now follow Quillen [Q2] to explain how to turn $\lambda$ into a smooth line bundle.

Take $y_{0} \in B$. Let $J$ be a finite dimensional subspace of $H_{-, y_{0}}^{\infty}$ which is transversal to $\operatorname{Im}\left(D_{+, y_{0}}\right)\left[H_{+, y_{0}}^{1}\right]$ in $H_{-, y_{0}}^{0}$. A possible choice for $J$ is $\operatorname{Ker} D_{-, y_{0}}$. Using the local triviality of $M \rightarrow B$, we can as well assume that $J$ is now a smooth subbundle of $H_{-, y_{0}}^{\infty}$ over an open set $U$ in $B$ containing $y_{0}$, such that the transversality assumption still holds at any $y \in U$. Since $D_{+}$is elliptic, $D_{+}^{-1} J \in H_{+}^{\infty}$.

Consider the exact sequence

$$
0 \rightarrow \operatorname{Ker} D_{+} \rightarrow D_{+}^{-1} J \underset{D_{+}}{\rightarrow} J \rightarrow \operatorname{Coker} D_{+} \rightarrow 0
$$

We can canonically identify $\lambda$ and $\operatorname{det}\left(D_{+}^{-1} J\right)^{*} \otimes \operatorname{det} J$ by the following construction. Take $s \neq 0$ in $\operatorname{det}\left(\operatorname{Ker} D_{+}\right), s^{\prime} \neq 0$ in $\operatorname{det}\left(\right.$ Coker $\left.D_{+}\right)$. $s$ can completed into $s \wedge \bar{s} \in \operatorname{det}\left(D_{+}^{-1} J\right)$ with $s \wedge \bar{s} \neq 0$. Similarly take $\bar{s}^{\prime}$ in $\Lambda^{\operatorname{dim} \text { Coker } D_{+}}(J)$ whose image in $\operatorname{det}\left(\operatorname{Coker} D_{+}\right)$is $s^{\prime}$.

Then $\quad s \otimes(s \wedge \bar{s})^{*} \otimes\left(\bar{s}^{\prime} \wedge D \bar{s}\right) \otimes s^{*} \quad$ is $\quad$ non-zero in $\quad \operatorname{det}\left(\operatorname{Ker} D_{+}\right) \otimes$ 
$\operatorname{det}\left(D_{+}^{-1} J\right)^{*} \otimes \operatorname{det} J \otimes\left(\text { Coker } D_{+}\right)^{*}$ and does not depend on $s, \bar{s}, s^{\prime}, \bar{s}^{\prime}$. We can thus identify $s^{*} \otimes s^{\prime} \in \lambda$ with $(s \wedge \bar{s})^{*} \otimes \bar{s}^{\prime} \wedge D \bar{s} \in \operatorname{det}\left(D_{+}^{-1} J\right)^{*} \otimes \operatorname{det} J .\left(\operatorname{det} D_{+}^{-1} J\right)^{*} \otimes$ $\operatorname{det} J$ is a smooth line bundle over $U$. If $J^{\prime}$ is another smooth subbundle of $H_{-}^{\infty}$ having the same properties as $J$, one easily verifies that when identifying $\left(\operatorname{det} D_{+}^{-1} J\right)^{*} \otimes \operatorname{det} J$ and $\left(\operatorname{det} D_{+}^{-1} J^{\prime}\right) \otimes \operatorname{det} J^{\prime}$ with $\lambda$, the transition maps are smooth.

$\lambda$ then becomes a smooth line bundle over $B$. We now proceed as in [Q2]. Clearly

$$
D^{2}=\left[\begin{array}{cc}
D_{-} D_{+} & 0 \\
0 & D_{+} D_{-}
\end{array}\right]
$$

The spectrum of $D^{2}$ is discrete, the non-zero eigenvalues of $D_{+} D_{-}$and $D_{-} D_{+}$agree, and the corresponding eigenspaces are mapped isomorphically by $D$. For $a>0$ not in the spectrum, let $K_{ \pm}^{a}$ be the sum of the eigenspaces for eigenvalues less than $a$. Then since $D^{2}$ is fiberwise elliptic, $K_{ \pm}^{a}$ consists of $C^{\infty}$ sections of $F_{ \pm} \otimes \xi$ over $Z$.

The exact sequence corresponding to (1.31) is now

$$
0 \rightarrow \operatorname{Ker} D_{+} \rightarrow K_{+}^{a} \underset{D_{+}}{\rightarrow} K_{-}^{a} \rightarrow \operatorname{Ker} D_{-} \rightarrow 0 \text {. }
$$

Set

$$
\lambda^{a}=\left(\operatorname{det} K_{+}^{a}\right)^{*} \otimes \operatorname{det} K_{-}^{a} .
$$

$K_{ \pm}^{a}$ are smooth finite dimensional subbundles of $H^{\infty}$ over the open set $U^{a}=$ $\left(a \notin \operatorname{Spec} D^{2}\right) . \lambda^{a}$ is then a smooth line bundle over $U^{a}$. We identify $\lambda$ and $\lambda^{a}$ over $U^{a}$ as before. For $a, b$ with $a<b$ not in the spectrum of $D^{2}$, let $K_{ \pm}^{(a, b)}$ be the union of the eigenspaces corresponding to eigenvalues $\mu$ with $a<\mu<b . K_{ \pm}^{(a, b)}$ are smooth subbundles of $H_{ \pm}^{\infty}$ over $U^{a} \cap U^{b}$. Set

$$
\lambda^{(a, b)}=\left(\operatorname{det} K_{+}^{(a, b)}\right)^{*} \otimes \operatorname{det} K_{-}^{(a, b)} .
$$

Let $D_{+}^{(a, b)}$ be the restriction of $D_{+}$to $K_{+}^{(a, b)}$. $D_{+}$maps $K_{+}^{(a, b)}$ into $K_{-}^{(a, b)}$.

Clearly, over $U^{a} \cap U^{b}$

$$
\lambda^{b}=\lambda^{a} \otimes \lambda^{(a, b)} .
$$

The identification of $\lambda^{a}$ and $\lambda^{b}$ via $\lambda$ is given by the mapping

$$
s \in \lambda^{a} \rightarrow s \otimes \operatorname{det} D_{+}^{(a, b)} \in \lambda^{b} .
$$

\section{g) Quillen Metrics on $\lambda$}

As subbundles of $H_{ \pm}^{\infty}$, the bundles $K_{ \pm}^{a}$ over $U^{a}$ or $K_{ \pm}^{(a, b)}$ over $U^{a} \cap U^{b}$ inherit the Hermitian product (1.3) of $H_{ \pm}^{\infty}$. The bundles $\lambda^{a}, \lambda^{(a, b)}$ are then naturally endowed with metrics ||$^{a},||^{(a, b)}$.

Over $U^{a} \cap U^{b}, K_{ \pm}^{a}$ is orthogonal to $K_{ \pm}^{(a, b)}$. It follows that if $s \in \lambda^{a}$,

$$
\left|s \otimes \operatorname{det} D_{+}^{(a, b)}\right|^{b}=|s|^{a}\left|\operatorname{det} D_{+}^{(a, b)}\right|^{(a, b)} .
$$

When identifying $\lambda$ with $\lambda^{a}$ or $\lambda^{b}$, the metrics ||$^{a}$ and ||$^{b}$ are related to each other by

$$
||^{b}=\left.1 \quad\right|^{a}\left|\operatorname{det} D_{+}^{(a, b)}\right|^{(a, b)}
$$


To correct this discrepancy, we will proceed as in Quillen [Q1], using a zêta function regularization of $\left|\operatorname{det} D_{+}\right|$.

Definition 1.8. Over $U^{a}, P^{a}$ is the orthogonal projection operator from $H^{\infty}=$ $H_{+}^{\infty} \oplus H_{-}^{\infty}$ on $K^{a}=K_{+}^{a} \oplus K_{-}^{a} \cdot Q^{a}$ is the operator

$$
Q^{a}=I-P^{a}
$$

Since $K_{ \pm}^{a}$ are smooth bundles in $H_{ \pm}^{\infty}, P^{a}$ is a smooth family of regularizing operators over $U^{a}$.

Definition 1.9. For $s \in C, a>0, y \in U^{a}$, set

$$
\zeta_{y}^{a}(s)=\frac{1}{2} \operatorname{Tr}\left[\left([D]^{2}\right)^{-s} Q^{a}\right]
$$

or equivalently

$$
\zeta_{y}^{a}(s)=\frac{1}{2 \Gamma(s)} \int_{0}^{+\infty} t^{s-1} \operatorname{Tr}\left[e^{-t D^{2}} Q^{a}\right] d t .
$$

$\zeta^{a}(s)$ is exactly the zeta function of the operator $D_{-} D_{+}$restricted to the eigenspaces whose eigenvalues are larger than $a$. Since $P^{a}$ is trace class, using (1.28), we find that as $t \downarrow \downarrow 0$,

$$
\frac{1}{2} \operatorname{Tr}\left[\exp \left(-\left(t D^{2}\right) Q^{a}\right]=\sum_{-n / 2}^{-1} A_{j} t^{j}+0(1, y) .\right.
$$

Also since $a>0, \operatorname{Tr}\left[e^{-t D^{2}} Q^{a}\right]$ decays exponentially and uniformly over compact subsets of $U^{a}$.

Using (1.38), we find that as is well-known (see Seeley [Se]), $\zeta^{a}(s)$ is holomorphic for Res $>n / 2$ and meromorphic on $C$. Moreover $\zeta^{a}$ is holomorphic at 0 and $\zeta_{y}^{a}(0)$ and $\partial \zeta_{y}^{a} / \partial s(0)$ are smooth in $y \in U^{a}$.

For $0<a<b<+\infty$, we can also define $\zeta^{(a, b)}(s)$. Clearly

$$
\zeta^{a}(s)=\zeta^{(a, b)}(s)+\zeta^{b}(s) .
$$

Also we have the trivial relation

$$
\left|\operatorname{det} D_{+}^{(a, b)}\right|^{(a, b)}=\exp \left\{-\frac{1}{2} \frac{\partial \zeta^{(a, b)}}{\partial s}(0)\right\} .
$$

$\mu$ is now a fixed real number.

Definition 1.10. \|\|$^{a}$ denotes the metric on $\lambda^{a}$ which is such that if $l \in \lambda^{a}$,

$$
\|l\|^{a}=|l|^{a} \exp \left\{-\frac{1}{2} \frac{\partial \zeta^{a}}{\partial s}(0)-\frac{1}{2} \mu A_{0}\right\}
$$

We now have the natural extension of Quillen [Q2].

Theorem 1.11. Under the canonical identification of $\lambda$ with $\lambda^{a}$ over $U^{a}$, the metrics $\|\quad\|^{a}$ patch into a smooth metric \|\| on $\lambda$ over $B$.

Proof. Using (1.35) and (1.39), the result is obvious. 
Remark 3. In Quillen [Q2], it turns out that $A_{0}$ is constant. Here the reader may ask why we introduce the factor $\mu A_{0}$ in (1.41). In fact for $b>0$, consider the new family $b D_{+}$. The new metric on $\lambda$ is now $b^{A_{0}}\|\| . A_{0}(y)$ should be thought of as the formal dimension of $H_{+, y}^{\infty}$. However since $A_{0}$ varies with $y$, this dimension is anomalous. The introduction of the parameter $\mu$ permits us to consider all the scaled metrics altogether.

\section{h) Construction of Connection Forms}

We temporarily assume that $0 \leqq a<b \leqq+\infty$. Let $P^{(a, b)}$ be the orthogonal projection operator on $K^{(a, b)}$. In particular $P^{(a,+\infty)}=Q^{a}$.

$P_{ \pm}^{(a, b)}$ is the restriction of $P^{(a, b)}$ to $K_{ \pm}^{(a, b)}$.

Definition $1.12 .{ }^{0} \nabla^{(a, b)}$ is the connection on $K_{ \pm}^{(a, b)}$ defined by the relation

$$
{ }^{0} \nabla^{(a, b)}=P^{(a, b)} \tilde{\nabla}^{u} \text {. }
$$

Since $\tilde{\nabla}^{u}$ is unitary on $H^{\infty},{ }^{0} \nabla^{(a, b)}$ is unitary on $K^{(a, b)}$. For $0<a<b<+\infty,{ }^{0} \nabla^{(a, b)}$ induces a connection on $\lambda^{(a, b)}$ which is unitary for ||$^{(a, b)}$. In the sequel, if $0<a<$ $+\infty$, over $U^{a}$, we write ${ }^{0} \nabla^{a}$ instead ${ }^{0} \nabla^{(0, a)}$.

We first prove a technical result.

Proposition 1.13. $\tilde{\nabla}^{u} P^{(a, b)}$ interchanges $K_{ \pm}^{(a, b)}$ and $K_{ \pm}^{(0, a)} \oplus K_{ \pm}^{(b,+\infty)}$. Also

$$
\begin{aligned}
{ }^{0} \nabla^{(a, b)} D^{(a, b)} & =P^{(a, b)}\left(\tilde{\nabla}^{u} D\right) P^{(a, b)}, \\
d \frac{1}{2} \operatorname{Tr}\left[\exp \left(-t D^{2}\right) P^{(a, b)}\right] & =-t \operatorname{Tr}\left[\exp \left(-t D^{2}\right) \tilde{\nabla}^{u} D D P^{(a, b)}\right] .
\end{aligned}
$$

Proof. Since $\left(P^{(a, b)}\right)^{2}=P^{(a, b)}$, we get

$$
\tilde{\nabla}^{u} P^{(a, b)}=P^{(a, b)} \tilde{\nabla}^{u} P^{(a, b)}+\tilde{\nabla}^{u} P^{(a, b)} P^{(a, b)} .
$$

The first part of the proposition is proved. Since $D$ commutes with $P^{(a, b)}$, the first line of (1.43) is obvious.

Also

$$
\begin{aligned}
d\left[\frac{1}{2} \operatorname{Tr} \exp \left(-t D^{2}\right) P^{(a, b)}\right]= & -t \operatorname{Tr}\left[\exp \left(-t D^{2}\right) \tilde{\nabla}^{u} D D P^{(a, b)}\right] \\
& +\frac{1}{2} \operatorname{Tr}\left[\exp \left(-t D^{2}\right) \tilde{\nabla}^{u} P^{(a, b)}\right] .
\end{aligned}
$$

By the first part of the proposition, the last term in the right-hand side of (1.45) is 0 . The proposition is proved.

Since $P^{a}$ is a smooth family of regularizing operators, using Theorem 1.7, we have the expansions as $t \downarrow \downarrow 0$,

$$
\begin{aligned}
\frac{1}{2} \operatorname{Tr}\left[\exp \left(-t D^{2}\right) Q^{a}\right] & =\sum_{-n / 2}^{-1} A_{j} t^{j}+0(1, y), \\
\operatorname{Tr}\left[\exp \left(-t D^{2}\right) \tilde{\nabla}^{u} D D Q^{a}\right] & =-\sum_{-n / 2}^{0} d A_{j} t^{j-1}+0(1, y), \\
\operatorname{Tr}_{s}\left[\exp \left(-t D^{2}\right) \tilde{\nabla}^{u} D D Q^{a}\right] & =-\sum_{-n / 2}^{0} B_{j} t^{j-1}+0(1, y) .
\end{aligned}
$$


Still $\operatorname{Tr}\left[\exp \left(-t D^{2}\right) \tilde{\nabla}^{u} D D Q^{a}\right]$ is real and $\operatorname{Tr}_{s}\left[\exp \left(-t D^{2}\right) \tilde{\nabla}^{u} D D Q^{a}\right]$ is purely imaginary.

The analogue of $(1.30)$ is now

$$
\begin{aligned}
\operatorname{Tr}\left[\exp \left(-t D_{+} D_{-}\right) \tilde{\nabla}^{u} D_{+} D_{-} P^{(a, b)}\right]= & \frac{1}{2} \operatorname{Tr}\left[\exp \left(-t D^{2}\right) \nabla^{u} D D P^{(a, b)}\right] \\
& -\frac{1}{2} \operatorname{Tr}_{s}\left[\exp \left(-t D^{2}\right) \tilde{\nabla}^{u} D D P^{(a, b)}\right] .
\end{aligned}
$$

We now define a family of one forms on $U^{a}$.

Definition 1.14. For $t>0, \gamma_{t}^{a}, \delta_{t}^{a}$ are the $C^{\infty}$ differential forms over $U^{a}$,

$$
\gamma_{t}^{a}=\int_{t}^{+\infty} \operatorname{Tr}\left[e^{-s D^{2}} \tilde{\nabla}^{u} D D Q^{a}\right] d s, \quad \delta_{t}^{a}=\int_{t}^{+\infty} \operatorname{Tr}_{s}\left[e^{-s D^{2}} \widetilde{\nabla}^{u} D D Q^{a}\right] d s
$$

Similarly for $0<a<b<+\infty, t \geqq 0, \gamma_{t}^{(a, b)}, \delta_{t}^{(a, b)}$ are the $C^{\infty}$ differential forms over $U^{a} \cap U^{b}$

$$
\begin{aligned}
& \gamma_{t}^{(a, b)}=\int_{t}^{+\infty} \operatorname{Tr}\left[e^{-s D^{2}} \widetilde{\nabla}^{u} D D P^{(a, b)}\right] d s, \\
& \delta_{t}^{(a, b)}=\int_{t}^{+\infty} \operatorname{Tr}_{s}\left[e^{-s D^{2}} \tilde{\nabla}^{u} D D P^{(a, b)}\right] d s .
\end{aligned}
$$

Theorem 1.15. For any $t>0$,

$$
\gamma_{t}^{a}=-\operatorname{Tr}\left[e^{-t D^{2}}[D]^{-1}\left(\tilde{\nabla}^{u} D\right) Q^{a}\right], \quad \delta_{t}^{a}=\operatorname{Tr}_{s}\left[e^{-t D^{2}}[D]^{-1}\left(\tilde{\nabla}^{u} D\right) Q^{a}\right] .
$$

As $t \downarrow \downarrow 0$, we have the expansions,

$$
\begin{aligned}
& \gamma_{t}^{a}=\sum_{-n / 2}^{-1} d A_{j} \frac{t^{j}}{j}+d A_{0} \log (t)+\gamma_{0}^{a}+0(t, y), \\
& \delta_{t}^{a}=\sum_{-n / 2}^{-1} B_{j} \frac{t^{j}}{j}+B_{0} \log (t)+\delta_{0}^{a}+0(t, y),
\end{aligned}
$$

where $\gamma_{0}^{a}, \delta_{0}^{a}$ are $C^{\infty} 1$-forms on $U^{a}$, which are respectively real and purely imaginary, and $0(t, y)$ is uniform on the compact subsets of $U^{a}$. Also the following identities hold:

$$
\begin{aligned}
& d \zeta^{a}(0)=d A_{0}, \quad d\left[\frac{\partial}{\partial s} \zeta^{a}(0)\right]=-\gamma_{0}^{a}-\Gamma^{\prime}(1) d A_{0}, \\
& \gamma_{0}^{a}+\Gamma^{\prime}(1) d A_{0}=-\left(s \operatorname{Tr}\left[\left(D^{2}\right)^{-s} D^{-1} \tilde{\nabla}^{u} D Q^{a}\right]\right)^{\prime}(0), \\
& \delta_{0}^{a}+\Gamma^{\prime}(1) B_{0}=\left(s \operatorname{Tr}_{s}\left[\left(D^{2}\right)^{-s} D^{-1} \tilde{\nabla}^{u} D Q^{a}\right]\right)^{\prime}(0), \\
& \frac{1}{2}\left[\left(\gamma_{0}^{a}-\delta_{0}^{a}\right)+\Gamma^{\prime}(1)\left(d A_{0}-B_{0}\right)\right]=-\left(s \operatorname{Tr}\left[\left(D_{-} D_{+}\right)^{-s}\left(D_{+}\right)^{-1} \tilde{\nabla}^{u} D_{+} Q^{a}\right]\right)^{\prime}(0) .
\end{aligned}
$$

$d A_{0}$ (respectively $-B_{0}$ ) is the residue at $s=0$ of

$$
\left.\operatorname{Tr}\left[\left(D^{2}\right)^{-s} D^{-1} \tilde{\nabla}^{u} D Q^{a}\right] \text { (respectively } \operatorname{Tr}_{s}\left[\left(D^{2}\right)^{-s} D^{-1} \tilde{\nabla}^{u} D Q^{a}\right]\right) .
$$

For $0<a<b<+\infty$, on $U^{a} \cap U^{b}$,

$$
\begin{aligned}
& \gamma_{0}^{a}=\gamma_{0}^{(a, b)}+\gamma_{0}^{b}, \quad \delta_{0}^{a}=\delta_{0}^{(a, b)}+\delta_{0}^{b}, \\
& \frac{1}{2}\left(\gamma_{0}^{(a, b)}-\delta_{0}^{(a, b)}\right)=\frac{{ }^{0} \nabla^{(a, b)} \operatorname{det} D_{+}^{(a, b)}}{\operatorname{det} D_{+}^{(a, b)}} .
\end{aligned}
$$


Proof. Using Proposition 1.13, we obtain easily the first part of (1.49) (with our sign conventions!).

Also for $t>0$,

$$
\begin{aligned}
\gamma_{t}^{a}= & \int_{t}^{1}\left[\operatorname{Tr}\left[e^{-s D^{2}} \tilde{\nabla}^{u} D D Q^{a}\right]+\sum_{-n / 2}^{0} d A_{j} s^{j-1}\right] d s \\
& +\int_{1}^{+\infty} \operatorname{Tr}\left[e^{-s D^{2}} \tilde{\nabla}^{u} D D Q^{a}\right] d s-\sum_{-n / 2}^{-1} \frac{d A_{j}}{j}+\sum_{j=-n / 2}^{-1} \frac{d A_{j}}{j} t^{j}+d A_{0} \log (t) .
\end{aligned}
$$

Using (1.45), we find that as $t \downarrow \downarrow 0$, the first integral in (1.54) has a limit. We thus obtain (1.50).

Also for $\operatorname{Re}(s)$ large enough,

$$
\begin{aligned}
d \zeta^{a}(s)= & -\frac{1}{\Gamma(s)} \int_{0}^{+\infty} t^{s} \operatorname{Tr}\left[e^{-t D^{2}} \tilde{\nabla}^{u} D D Q^{a}\right] d t \\
= & -\frac{1}{\Gamma(s)} \int_{0}^{1} t^{s}\left(\operatorname{Tr}\left[e^{-t D^{2}} \tilde{\nabla}^{u} D D Q^{a}\right]+\sum_{-n / 2}^{0} d A_{j} t^{j-1}\right) d t \\
& -\frac{1}{\Gamma(s)} \int_{1}^{+\infty} t^{s} \operatorname{Tr}\left[e^{-t D^{2}} \tilde{\nabla}^{u} D D Q^{a}\right] d t+\frac{1}{\Gamma(s)} \sum_{j=-n / 2}^{-1} \frac{d A_{j}}{s+j}+\frac{d A_{0}}{\Gamma(s+1)} .
\end{aligned}
$$

The first equation of (1.51) now follows from (1.46) and (1.55). When comparing (1.54) and (1.55) we obtain the second equation of (1.51).

By Atiyah-Patodi-Singer [APS1, Proposition 2.9], we know that

$$
d \zeta^{a}(s)=-s \operatorname{Tr}\left[\left(D^{2}\right)^{-s-1} \tilde{\nabla}^{u} D D Q^{a}\right]=s \operatorname{Tr}\left[\left(D^{2}\right)^{-s} D^{-1} \tilde{\nabla}^{u} D Q^{a}\right] .
$$

We thus obtain the third equation of (1.51).

The fourth equality in (1.51) can be proved by proceeding as in (1.54), (1.55). The end of (1.51) is trivial. Using (1.55), we find that $d A_{0}$ is the residue at $s=0$ of $d \zeta^{a}(s) / s$. The result on $B_{0}$ can be proved by still proceeding as in (1.55).

The first two equations of (1.53) are trivial using (1.47). Also by the obvious analogue of (1.49) for $\gamma_{t}^{(a, b)}$ which is valid at $t=0$, we have

$$
\begin{aligned}
\frac{1}{2}\left(\gamma_{0}^{(a, b)}-\delta_{0}^{(a, b)}\right)= & -\frac{1}{2} \operatorname{Tr}\left[\left[D^{(a, b)}\right]^{-10} \nabla^{(a, b)} D^{(a, b)}\right] \\
& -\frac{1}{2} \operatorname{Tr}_{s}\left[\left[D^{(a, b)}\right]^{-10} \nabla^{(a, b)} D^{(a, b)}\right] \\
= & -\operatorname{Tr}\left[\left(D_{+}^{(a, b)}\right)^{-10} \nabla^{(a, b)} D_{+}^{(a, b)}\right] .
\end{aligned}
$$

With our sign conventions, we clearly have

$$
\frac{{ }^{0} \nabla^{(a, b)} \operatorname{det} D_{+}^{(a, b)}}{\operatorname{det} D_{+}^{(a, b)}}=-\operatorname{Tr}\left[\left[D_{+}^{(a, b)}\right]^{-10} \nabla^{(a, b)} D_{+}^{(a, b)}\right] .
$$

The theorem is proved.

Remark 4. (1.52) shows that

$$
\operatorname{Tr}\left[\left(D^{2}\right)^{-s} D^{-1} \tilde{\nabla}^{u} D Q^{a}\right]=\frac{d A_{0}}{s}+\varphi^{a}(s), \quad \operatorname{Tr}_{s}\left[\left(D^{2}\right)^{-s} D^{-1} \tilde{\nabla}^{u} D Q^{a}\right]=-\frac{B_{0}}{s}+\psi^{a}(s),
$$


where $\varphi^{a}, \psi^{a}$ are holomorphic at $s=0$, and that moreover

$$
\gamma_{0}^{a}+\Gamma^{\prime}(1) d A_{0}=-\varphi^{a}(0), \quad \delta_{0}^{a}+\Gamma^{\prime}(1) B_{0}=\psi^{a}(0) .
$$

Also observe that if the family $D$ is replaced by $b D$ with $b>0, d A_{0}, B_{0}$ do not vary, but $\gamma_{0}^{a}, \delta_{0}^{a}$ are changed into $\gamma_{0}^{a}+2 d A_{0} \log b, \delta_{0}^{a}+2 B_{0} \log b$. In view of (1:51), this again appears as a scaling discrepancy. This problem will be considered in more detail in [BF2].

\section{i) Additivity Property of Quillen's Superconnections}

Let $D^{a}$ be the restriction of $D$ to $K^{a}$. Over $U^{a}$, the $Z_{2}$ graded bundle $K^{a}=K_{+}^{a} \oplus K_{-}^{a}$ is endowed with the superconnection ${ }^{0} \nabla^{a}+\sqrt{ } t D^{a}$. We now relate this superconnection to the superconnection $\tilde{\nabla}^{u}+\sqrt{ } t D$.

Theorem 1.16. On $U^{a}$, the following identity holds:

$$
\begin{aligned}
{\left[\operatorname{Tr}_{s} \exp -\left(\tilde{\nabla}^{u}+\sqrt{ } t D\right)^{2}\right]^{(2)}=} & \operatorname{Tr}_{s}\left[\exp -\left({ }^{0} \nabla^{a}+\sqrt{ } t D^{a}\right)^{2}\right]^{(2)} \\
& +\operatorname{Tr}_{s}\left[\exp -\left({ }^{0} \nabla^{(a,+\infty)}+\sqrt{ } t D^{(a,+\infty)}\right)^{2}\right]^{(2)} .
\end{aligned}
$$

Proof. $H_{ \pm}^{\infty}$ splits into

$$
H_{ \pm}^{\infty}=K_{ \pm}^{a} \oplus K_{ \pm}^{(a,+\infty)} .
$$

Let $\tilde{\nabla}^{\prime}$ be the connection on $H^{\infty}$ which preserves the splitting $\tilde{\nabla}^{\prime}={ }^{0} \nabla^{a} \oplus{ }^{0} \nabla^{(a,+\infty)}$. Set

$$
M^{a}=\tilde{\nabla}^{u}-\tilde{\nabla}^{\prime} .
$$

$M^{a}$ is a one form with values in End $H_{ \pm}^{\infty}$. Recall that by Proposition 1.13, $\tilde{\nabla}^{u} P_{ \pm}^{a}$ interchanges $K_{ \pm}^{a}$ and $K_{ \pm}^{(a,+\infty)}$. We claim that with respect to the splitting (1.62), we have

$$
M^{a}=\left(\begin{array}{cc}
0 & -\tilde{\nabla}^{u} P^{a} \\
\tilde{\nabla}^{u} P^{a} & 0
\end{array}\right) .
$$

In fact if $h$ is a section of $K_{ \pm}^{a}$,

$$
\tilde{\nabla}^{u} h=\tilde{\nabla}^{u}\left(P^{a} h\right)=\left(\tilde{\nabla}^{u} P^{a}\right) h+P^{a} \tilde{\nabla}^{u} h .
$$

With respect to the splitting (1.62), for any $Y \in T B, M^{a}(Y)$ is odd, and so

$$
\operatorname{Tr}_{s} M^{a} e^{-t D^{2}}=0 \text {. }
$$

By proceeding as in (1.24), we have

$$
\frac{\partial}{\partial l} \operatorname{Tr}_{s}\left[\exp -\left(\tilde{\nabla}^{u}+l M^{a}+\sqrt{ } t D\right)^{2}\right]=-d \operatorname{Tr}_{s}\left[M^{a} \exp -\left(\tilde{\nabla}^{u}+l M^{a}+\sqrt{ } t D\right)^{2}\right] .
$$

Since $M^{a}$ is of degree 1 in the Grassmann variables, we get from (1.66),

$$
\left(\frac{\partial}{\partial l} \operatorname{Tr}_{s}\left[\exp -\left(\tilde{\nabla}^{u}+l M^{a}+\sqrt{ } t D\right)^{2}\right]\right)^{(2)}=0 .
$$

(1.61) is proved. 


\section{j) A Unitary Connection on $\lambda$}

We are now ready to define a unitary connection on $\lambda$.

Definition 1.17. ${ }^{1} \nabla^{a}$ denotes the connection on $\lambda^{a}$ over $U^{a}$ given by

$$
{ }^{1} \nabla^{a}={ }^{0} \nabla^{a}+\frac{1}{2}\left(\gamma_{0}^{a}-\delta_{0}^{a}\right)+\frac{1}{2}\left(\Gamma^{\prime}(1)-\mu\right)\left(d A_{0}-B_{0}\right) .
$$

We now prove the fundamental result of this section.

Theorem 1.18. Identifying $\lambda$ with $\lambda^{a}$ over $U^{a}$, the connections ${ }^{1} \nabla^{a}$ patch together into a connection ${ }^{1} \nabla$ on $\lambda$, which is unitary for the metric \|\| . The curvature of ${ }^{1} \nabla$ is given by $a_{0}^{(2)}$.

Proof. Recall that ${ }^{0} \nabla^{a}$ is unitary on $\left(\lambda^{a},||^{a}\right)$. To check that ${ }^{1} \nabla^{a}$ is unitary on $(\lambda,\|\|)$, we can disregard $\delta_{0}^{a}$ and $B_{0}$ which are purely imaginary. Using the second line of (1.51), it is clear that ${ }^{1} \nabla^{a}$ is unitary with respect to \|\| .

Take $0<a<b<+\infty$. Let $l$ be a smooth section of $\lambda^{a}$ over $U^{a} \cap U^{b}$. Clearly

$$
{ }^{0} \nabla^{b}\left(l \otimes \operatorname{det} D_{+}^{(a, b)}\right)={ }^{0} \nabla^{a} l \otimes \operatorname{det} D_{+}^{(a, b)}+l \otimes{ }^{0} \nabla^{(a, b)} \operatorname{det} D_{+}^{(a, b)} .
$$

Using the last equality in (1.53), we get

$$
{ }^{0} \nabla^{b}\left(l \otimes \operatorname{det} D_{+}^{(a, b)}\right)=\left({ }^{0} \nabla^{a}+\frac{1}{2}\left(\gamma_{0}^{(a, b)}-\delta_{0}^{(a, b)}\right)\right) l \otimes \operatorname{det} D_{+}^{(a, b)} .
$$

Using the first two equalities in (1.53), we find that

$$
{ }^{1} \nabla^{b}\left(l \otimes \operatorname{det} D_{+}^{(a, b)}\right)=\left({ }^{1} \nabla^{a} l\right) \otimes \operatorname{det} D_{+}^{(a, b)} .
$$

Using (1.72), we find that the connections ${ }^{1} \nabla^{a}$ patch together.

We now use equality (1.61). Clearly since $K^{a}$ is finite dimensional,

$$
\lim _{t \downarrow \downarrow 0}\left[\operatorname{Tr}_{s} \exp -\left({ }^{0} \nabla^{a}+\sqrt{ } t D\right)^{2}\right]^{(2)}=\left[\operatorname{Tr}_{s} \exp -\left({ }^{0} \nabla^{a}\right)^{2}\right]^{(2)}=-\operatorname{Tr}_{s}\left[{ }^{0} \nabla^{a}\right]^{2}
$$

An easy extension of Theorem 1.6, shows that for $0<t<T<+\infty$,

$$
\begin{aligned}
& \operatorname{Tr}_{s} \exp -\left({ }^{0} \nabla^{(a,+\infty)}+\sqrt{ } t D^{(a,+\infty)}\right)^{2}-\operatorname{Tr}_{s} \exp -\left({ }^{0} \nabla^{(a,+\infty)}+\sqrt{ } T D^{(a,+\infty)}\right)^{2} \\
& \quad=-\frac{d}{2} \int_{t}^{T} \operatorname{Tr}_{s}\left[\exp \left(-s D^{2}\right) \tilde{\nabla}^{u} D D Q^{a}\right] d s .
\end{aligned}
$$

Since $a$ is $>0$, as $T \uparrow+\infty \operatorname{Tr}_{s}\left[\exp -\left({ }^{0} \nabla^{(a,+\infty)}+\sqrt{ } T D^{(a,+\infty)}\right)^{2}\right]$ decays exponentially. We find that

$$
\operatorname{Tr}_{s} \exp -\left({ }^{0} \nabla^{(a,+\infty)}+\sqrt{ } t D^{(a,+\infty)}\right)^{2}=-\frac{d}{2} \delta_{t}^{a} .
$$

By differentiating the second line of (1.50), we find that as $t \downarrow \downarrow 0$,

$$
d \delta_{t}^{a}=\sum_{-n / 2}^{-1} d B_{j} \frac{t^{j}}{j}+d B_{0} \log t+d \delta_{0}^{a}+0(t, y) .
$$

Using (1.14), (1.73), (1.75), (1.76) and identifying the coefficients in the expansions of both sides of (1.61), we get 


$$
a_{0}^{(2)}=-\operatorname{Tr}_{s}\left[{ }^{0} \nabla^{a}\right]^{2}-\frac{d}{2} \delta_{0}^{a}
$$

Now $-\operatorname{Tr}_{s}\left[{ }^{0} \nabla^{a}\right]^{2}$ is exactly the curvature of $\lambda^{a}$ for the connection ${ }^{0} \nabla^{a}$. Also by (1.51), $d \gamma_{0}^{a}=0$. Using (1.77), and the fact that $d B_{0}=0$, we find that the curvature of ${ }^{1} \nabla^{a}$ is $a_{0}^{(2)}$.

\section{k) Holomorphic Properties of ${ }^{1} \nabla$}

In [Q2], Quillen constructed a unitary holomorphic connection on the determinant bundle of a family of $\partial$ operators over a Riemann surface.

We now will prove that under the assumptions of [Q2], our connection coincides with Quillen's connection by proving that ${ }^{1} \nabla$ is holomorphic. As in [Q2] we will work in a product situation.

To simplify the notations, we now assume that $B=\mathbb{C}$ and that $M=Z \times \mathbb{C}$. $H_{y}^{\infty}$ is now a constant bundle over $\mathbb{C}$. We assume that $D_{+}$depends holomorphically on $y \in \mathbb{C}$, and $\tilde{\nabla}_{\partial / \partial y}^{u}, \tilde{\nabla}_{\partial / \partial \bar{y}}^{u}$ are the operators $\partial / \partial y, \partial / \partial \bar{y}$.

Let $J$ be a finite dimensional subspace of $H_{y_{0},-}^{\infty}$ which is transversal to Im $D_{+, y_{0}}\left[H_{+, y_{0}}^{1}\right] .\left(\operatorname{det} D_{+}^{-1} J\right)^{*} \otimes \operatorname{det} J$ is holomorphic on a neighborhood of $y_{0} \cdot \lambda$ inherits the corresponding holomorphic structure. $D_{-, y}$ is antiholomorphic in $y$. The eigenspaces $K^{a}$ are not holomorphic bundles. However $\lambda^{a}$, which is canonically isomorphic to $\lambda$ inherits the corresponding holomorphic structure.

Take $y_{0} \in U^{a}$. Set $J=K_{-, y_{0}}^{a} . V$ is a small neighborhood of $y_{0}$ in $U^{a}$ such that $P_{-}^{a}$ is one to one from $J$ into $K_{-, y}^{a}$ when $y \in V$. Then $P_{+}^{a}$ is one to one from $D_{+}^{-1} J$ into $K_{+}^{a}$. In fact if $x \in D_{+}^{-1} J$ and $P_{+}^{a} x=0$, then

$$
P_{-}^{a} D_{+} x=D_{+} P_{+}^{a} x=0
$$

and so $D_{+} x=0$, i.e. $x \in \operatorname{Ker} D_{+}$. Then $x=P_{+}^{a} x=0$. Since $P_{+}^{a}$ is one to one, if $m \in\left(\operatorname{det} D_{+}^{-1} J\right)^{*}, P_{+}^{a} m \in\left(\operatorname{det} K_{+}^{a}\right)^{*}$ is well-defined.

Proposition 1.19. Over $V$, the mapping

$$
m \otimes m^{\prime} \in\left(\operatorname{det} D_{+}^{-1} J\right)^{*} \otimes \operatorname{det} J \rightarrow P_{+}^{a} m \otimes P_{-}^{a} m^{\prime} \in \lambda^{a}
$$

is the canonical isomorphism of $\left(\operatorname{det} D_{+}^{-1} J\right)^{*} \otimes \operatorname{det} J$ and $\lambda^{a}$ via $\lambda$.

Proof. We take $s, \bar{s}, s^{\prime}, \bar{s}^{\prime}$ as in f). We can here assume that $\bar{s}^{\prime} \in \operatorname{det}\left(\operatorname{Ker} D_{-}\right)$. Clearly

$$
P_{+}^{a}(s \wedge \bar{s})=s \wedge P_{+}^{a} \bar{s}, \quad P_{-}^{a}\left(\bar{s}^{\prime} \wedge D_{+} \bar{s}\right)=\bar{s}^{\prime} \wedge D_{+}\left(P_{+}^{a} \bar{s}\right) .
$$

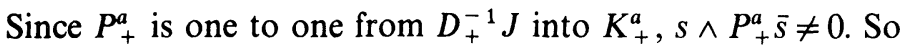

$$
P_{+}^{a}(s \wedge \vec{s})^{*} \otimes P_{-}^{a}\left(\vec{s}^{\prime} \wedge D_{+} \vec{s}\right)=\left(s \wedge P_{+}^{a} \vec{s}\right)^{*} \otimes \vec{s}^{\prime} \wedge D_{+}\left(P_{+}^{a} \vec{s}\right) .
$$

Using the canonical identifications with $\lambda$ given in $\mathrm{f}$ ), the proposition follows.

The second key step is the following:

Proposition 1.20. The connection ${ }^{0} \nabla^{a}$ on $\lambda^{a}$ is holomorphic.

Proof. Clearly $\partial / \partial \bar{y} D_{+}=0$. We now must prove that if $h$ is a holomorphic section of 
$D_{+}^{-1} J \otimes J$ over $V$, then

$$
{ }^{0} \nabla_{\partial / \partial \bar{y}}^{a}\left(P_{+}^{a} \otimes P_{-}^{a}\right)(h)=0
$$

Clearly

$$
\begin{aligned}
{ }^{0} \nabla_{\partial / \partial \bar{y}}^{a}\left(P_{+}^{a} \otimes P_{-}^{a}\right)(h)= & \left\{-\operatorname{Tr}_{K_{+}^{a}}\left[P_{+}^{a} \frac{\partial}{\partial \bar{y}} P_{+}^{a}\left(P_{+}^{a}\right)^{-1}\right]\right. \\
& \left.+\operatorname{Tr}_{K_{-}^{a}}\left[P_{-}^{a} \frac{\partial}{\partial \bar{y}} P_{-}^{a}\left(P_{-}^{a}\right)^{-1}\right]\right\}\left(P_{+}^{a} \otimes P_{-}^{a}\right)(h) .
\end{aligned}
$$

In (1.82), $\left(P^{a}\right)^{-1}$ denotes the inverse of $P^{a}$ restricted to $D_{+}^{-1} J$ or $J$.

Since $P_{-}^{a} D_{+}=D_{+} P_{+}^{a}$, we find $\left((\partial / \partial \bar{y}) P_{-}^{a}\right) D_{+}=D_{+}(\partial / \partial \bar{y}) P_{+}^{a}$, and so on $K_{+}^{a}$,

$$
P_{-}^{a}\left(\frac{\partial}{\partial \bar{y}} P_{-}^{a}\right)\left(P_{-}^{a}\right)^{-1} D_{+}=D_{+} P_{+}^{a}\left(\frac{\partial}{\partial \bar{y}} P_{+}\right)\left(P_{+}^{a}\right)^{-1} \text {. }
$$

Let $P_{\mathrm{Ker} D_{ \pm}}$be the orthogonal projection operator on $\operatorname{Ker} D_{ \pm}$. Using (1.83) and the fact that $D^{a}$ is one to one from $\left[\operatorname{Ker} D_{+}\right]^{\perp}$ into $\left[\operatorname{Ker} D_{-}\right]^{\perp}$, we find that the difference of traces appearing in (1.82) is given by

$$
-\operatorname{Tr}_{\mathrm{Ker} D_{+}}\left[P_{\mathrm{Ker} D_{+}} \frac{\partial}{\partial \bar{y}} P_{+}^{a}\left(P_{+}^{a}\right)^{-1}\right]+\operatorname{Tr}_{\mathrm{Ker} D_{-}}\left[P_{\mathrm{Ker} D_{-}} \frac{\partial}{\partial \bar{y}} P_{-}^{a}\left(P_{-}^{a}\right)^{-1}\right] .
$$

Now $\left(P_{ \pm}^{a}\right)^{-1}$ is the identity on $\operatorname{Ker} D_{ \pm}$. Also by Proposition $1.13, \partial / \partial \bar{y} P^{a}$ sends $K^{a}{ }_{ \pm}$in its orthogonal, and so

$$
P_{\mathrm{Ker} D_{ \pm}}\left(\frac{\partial}{\partial \bar{y}} P_{ \pm}^{a}\right) P_{\mathrm{Ker} D_{ \pm}}=0
$$

Equation (1.83) is 0 . The proposition is proved.

We finally obtain

Theorem 1.21. The connection ${ }^{1} \nabla$ is the unique holomorphic connection on $\lambda$ preserving \|\| .

Proof. Since $\partial / \partial \bar{y} D_{+}=0$, using (1.51), we find

$$
\left[\frac{1}{2}\left(\gamma_{0}^{a}-\delta_{0}^{a}\right)+\Gamma^{\prime}(1)\left(d A_{0}-B_{0}\right)\right]\left(\frac{\partial}{\partial \bar{y}}\right)=0
$$

Similarly, by (1.59) one finds easily that

$$
\left(d A_{0}-B_{0}\right)\left(\frac{\partial}{\partial \bar{y}}\right)=0 \text {. }
$$

Since ${ }^{0} \nabla^{a}$ is holomorphic on $\lambda^{a},{ }^{1} \nabla$ is also holomorphic. The theorem is proved.

Remark 5. On complex manifolds, the Dirac operator is given by $D=\bar{\partial}+\bar{\partial}^{*}$, and so in general $D_{+}$cannot be embedded in a holomorphic family.

However in the case considered by Quillen [Q2] where the fibers have complex dimension $1, D_{+}=\bar{\partial}$, and so $D_{+}$can depend holomorphically on a parameter. 
Acknowledgements. The authors are very grateful to Professors M.F. Atiyah, D. Quillen, I. Singer and E. Witten for useful conversations. They are also indebted to a referee for helpful comments.

\section{References}

[ABP] Atiyah, M. F., Bott, R., Patodi, V. K.: On the heat equation and the Index Theorem. Invent. Math. 19, 279-330 (1973)

[APS1] Atiyah, M. F., Patodi, V. K., Singer, I. M.: Spectral asymmetry and Riemannian Geometry, III. Math. Proc. Camb. Phil. Soc. 79, 71-99 (1976)

[AS1] Atiyah, M. F., Singer, I. M.: The Index of elliptic operators. IV. Ann. Math. 93, 119-138 (1971)

[B1] Bismut, J. M.: The Atiyah-Singer index theorem for families of Dirac operators: Two heat equation proofs. Invent. Math. 83, 91-151 (1986)

[BF1] Bismut, J. M., Freed D. S.: Fibré déterminant et invariant êta. C.R. Acad. Sci. Sér. 1. 301, 707$710(1985)$

[BF2] Bismut, J. M., Freed, D. S.: The analysis of elliptic families. Part II. (To appear in Commun. Math. Phys.)

[F] Freed, D. S.: (To appear)

[Gr] Greiner, P.: An asymptotic expansion for the heat equation. Arch. Ration. Mech. Anal. 41, 163218 (1971)

[Q1] Quillen, D.: Superconnections and the Chern character. Topology 24, 89-95 (1985)

[Q2] Quillen, D.: Determinants of Cauchy-Riemann operators over a Riemann surface. Funct. Anal. Appl. 19, 31-34 (1985)

[Se] Seeley, R. T.: Complex powers of an elliptic operator. Proc. Symp. Pure Math. AMS 10, 288307 (1967)

Communicated by A. Jaffe

Received December 24, 1985; in revised form February 13, 1986 